\title{
Increase in detection of Corynebacterium diphtheriae in Canada: 2006-2019
}

\author{
KA Bernard ${ }^{1,2 \star}$, AL Pacheco ${ }^{1}$, T Burdz $^{1}$, D Wiebe $^{1}$
}

\begin{abstract}
Background: Increasingly, matrix-assisted laser desorption/ionization time-of-flight mass spectrometry (MALDI-TOF) has been used to provide rapid, inexpensive and precise identification of bacteria, including Corynebacterium species. Only three Corynebacterium species are able to produce diphtheria toxin (DT), and strains recovered may be either toxin-producing or non-toxin-producing. It appears the more precise bacterial identification provided by MALDI-TOF systems has led to an increase in requests submitted to the National Microbiology Laboratory (NML) for toxin testing.

Objective: To describe the number of isolates identified as C. diphtheriae, C. ulcerans and C. pseudotuberculosis, submitted to the NML between January 2006 and July 30, 2019, including their geographic area, source, and whether they produce DT.
\end{abstract}

Methods: Referrals to the NML of human or animal isolates that were identified as any of those three Corynebacterium species were studied with respect to province, source and toxigenicity. Species identification was confirmed and then specimens were tested by polymerase chain reaction for the presence of tox genes and, if positive, for expression of DT by the modified Elek method. Analysis was descriptive.

Results: Over the study period, 639 isolates were identified as C. diphtheriae, 22 isolates as C. ulcerans; no isolates were identified as $C$. pseudotuberculosis. There was an increase in C. diphtheriae referrals for DT testing: from eight per year in 2006 to an average of 15 per month in 2019 , or a $1,200 \%$ increase over the 13.6 -year period. The referrals were primarily from western Canada ( $n=609 / 639 ; 95 \%)$. Most (638/639, 99\%) were human isolates and most were obtained from cutaneous sites. Of those isolates, $87 / 639$ (13.6\%) were found to be toxigenic and 552/639 (86.4\%) non-toxigenic. Among C. ulcerans referrals, $17 / 22$ (77\%) were from humans and five (23\%) were from animals, with 10/22 (45\%) being toxigenic.

Conclusion: There has been a marked increase in referrals to the NML for DT testing of Corynebacterium species. This could be due to the enhanced ability to identify these bacteria using MALDI-TOF systems. Ongoing monitoring will help to assess whether the increase is due solely to increased precision of diagnosis or whether these are emerging cutaneous pathogens.
This work is licensed under a Creative Commons Attribution 4.0 Internationa License.

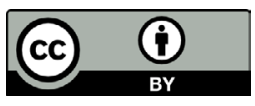

Affiliations

${ }^{1}$ National Microbiology Laboratory, Public Health Agency of Canada, Winnipeg, MB

${ }^{2}$ Department of Medical Microbiology, University of Manitoba, Winnipeg, MB

*Correspondence:

kathy.bernard@canada.ca

Suggested citation: Bernard KA, Pacheco AL, Burdz T, Wiebe D. Increase in detection of Corynebacterium diphtheriae in Canada: 2006-2019. Can Commun Dis Rep 2019;45(11):296-301.

https://doi.org/10.14745/ccdr.v45i11a04

Keywords: Corynebacterium diphtheriae, diphtheria-toxin-producing species, increase in recovery, MALDI-TOF

\section{Introduction}

Classic Corynebacterium diphtheriae (C. diphtheriae) infectioncausing sore throat, fever and respiratory symptoms-is rare in Canada (1-3). Since vaccination against diphtheria toxin (DT) is now part of the routine immunization schedule, there are fewer than four cases of this notifiable disease annually $(4,5)$.
In the past, Corynebacterium species isolated from non-respiratory clinical specimens were understudied and underreported. These bacteria were nearly always considered as contaminants, and were neither identified (i.e. genus and species) nor tested for antimicrobial susceptibility, even if recovered in pure culture from sterile body fluids or deep 
wound abscesses (3). As well, unambiguous identification of strains to genus and species could be difficult and expensive to perform solely using phenotypic test methods $(1,3)$.

It is now more widely appreciated that non-respiratory C. diphtheriae can cause skin and wound infections. Cutaneous diphtheria often presents as well-demarcated, sometimes foul-smelling ulcers or as nodules that are slow healing and highly contagious. People who are particularly at risk include those with co-morbidities such as HIV, hepatitis B or C, diabetes, a history of recurrent ulcers, alcohol abuse, a history of intravenous drug use, living in poorer-socioeconomic conditions, homeless shelters or refugee camps, or who travelled to countries where these pathogens are endemic (6-11). Treatment for cutaneous diphtheria often includes the use of the same systemic antibiotics as those used for respiratory disease, plus the implementation of isolation precautions $(6-10)$ to prevent bacteria from lesions serving as a reservoir for further spread of infection $(11,12)$. However, in some instances, difficult-to-treat multidrug resistant strains of $C$. diphtheriae have been detected from cases of cutaneous diphtheria (13). Occasionally, sepsis and death may result. The role of prior vaccination with diphtheria toxoid in preventing cutaneous infections is not well understood. The use of antitoxin is generally not recommended, unless signs of systemic toxicity are present (7).

Identification of the genus and species of Corynebacterium species has now been simplified by the widespread use of matrix-assisted laser desorption/ionization time-of-flight mass spectrometry (MALDI-TOF) instruments and more precise molecular methods. Proteins are extracted from bacteria and the resulting fingerprints are then compared to entries housed in the system's data library. High confidence identification of species can be obtained within a few minutes, at a cost of less than $\$ 1.00$ Canadian per sample $(14,15)$. The MALDI-TOF procedure has made it possible to routinely identify the diphtheria-toxin-producing species: $C$. diphtheriae; C. ulcerans; and $C$. pseudotuberculosis. Infections involving C. ulcerans and C. pseudotuberculosis are rare in humans and are acquired through infected animals. The $C$. ulcerans infections in humans have been linked to contact with diseased pets, such as dogs or cats, whereas C. pseudotuberculosis occurs in sheep and goats, so veterinarians or animal handlers are at increased risk for acquisition (3).

In Canada, the use of MALDI-TOF instruments began about 2012. These instruments are now widely distributed across the country-in all provincial public health laboratories, in many private/public hospital institutions and in veterinary laboratories specializing in infectious diseases of animals. The clear benefits for routine use of MALDI-TOF systems are the significantly reduced costs for characterizing bacteria and the greatly improved speed at which pathogens such as $C$. diphtheriae can be identified. These benefits result in prompt implementation of appropriate treatment options. However, MALDI-TOF systems can only identify these bacteria and cannot determine whether or not any of these Corynebacterium species actually produce DT. Over the past few years, the National Microbiology Laboratory (NML) began to receive an increasing number of requests for DT testing from public health laboratories and veterinary laboratories across the country. The objective of this study was to describe the number and features of the C. diphtheriae, C.ulcerans and C. pseudotuberculosis samples submitted to the NML between January 2006 and July 30, 2019, including the percentage that produced DT.

\section{Methods}

\section{Record review}

The NML's Special Bacteriology Unit (SBU) laboratory records were reviewed from 2006 to July 30, 2019 for test requests for DT detection. Information reviewed here included province, site of infection if known, the species of DT-producing Corynebacterium involved and whether or not the strain was toxigenic. Referrals received from January to July 2019 were further analyzed with respect to the source of the strain, as these data were typical of referrals in other years. Information regarding vaccination history and underlying patient history was generally scant or not provided.

\section{Overview of specimen types received}

Since 2011, Cadham Provincial Laboratory (Manitoba's public health laboratory) only forwards strains to the NML that have been found to be positive for tox gene targets by polymerase chain reaction (PCR) (D. Alexander, personal communication, July 24, 2019). Other provincial centres forward all strains that may require testing for $\mathrm{DT}$ to the $\mathrm{NML}$, as toxin analyses are not offered in their own laboratory settings. The NML receives pure cultures of bacterial isolates that have been recovered at provincial public health laboratories or by their clientele, which the provincial public health laboratories then forward to the NML for toxin testing. Occasionally strains are received from veterinary laboratories.

\section{Confirmation of Corynebacterium diphtheriae isolates}

From 2006 to approximately 2018, SBU strains had been tested phenotypically using an API CORYNE (bioMérieux, France) panel to provide species-level identification and to assist with categorizing the isolate with respect to one of the four $C$. diphtheriae biovars (i.e. biotypes: gravis; mitis; belfanti; and intermedius) $(1,3)$. The API CORYNE panel unambiguously identified $C$. diphtheriae for strains previously characterized by the gold standard 16S rRNA gene sequencing method; molecular methods are otherwise used at the NML to corroborate species level identification as required. The NML ceased characterization of strains to biovar as of May 2019, except upon request. Corynebacterium diphtheriae identification 
was validated for use by the NML for the Bruker Microflex and Biotyper library, where this method also provides unambiguous identification of species (data not shown).

\section{Identification of Corynebacterium ulcerans and C. pseudotuberculosis isolates}

These species are readily discernable from all others in the genus Corynebacterium, including $C$. diphtheriae, but are otherwise not easily differentiated from each other by commonly-used laboratory methods (1). Phenotypic results for these species, using conventional sugars or API CORYNE panels, are very similar $(1,2)$. These species have $99.4 \%$ similarity to each other by $16 \mathrm{~S}$ rRNA gene sequencing, which is too close to provide definitive discrimination from each other, and can produce similar scores to each other using MALDI-TOF systems (data not shown) (1). Therefore, precise identification of these species is corroborated at the NML by use of partial rpoB gene sequencing (16).

\section{Detection of diphtheria tox gene targets and expression of diphtheria toxin}

The SBU had used conventional PCR approaches to detect tox gene targets (13), but switched to a real time PCR-based method in 2018. Real-time PCR detects the tox gene and a region of the rpoB gene specific for $C$. diphtheriae using a Quant Studio platform (Applied Biosystems) $(17,18)$. The toxin-producing strain, C. diphtheriae ATCC 19409 (NCTC 3984), and the non-toxigenic strain, C. xerosis ATCC $373^{\top}$, were used as positive and negative controls, respectively. Expression of DT was detected by performing the modified Elek test only for strains where tox genes had been detected by PCR $(19,20)$. Elek-positive results may be reported at 48 hours, or as soon as expression is visually detected, with negative results being reportable after 48 hours. Isolates that are found to be positive for tox gene by PCR but negative for DT expression by Elek are reported as non-toxigenic (20); these occur rarely in Canada, accounting for $8 \%$ or fewer of all tox gene-positive strains (21).

\section{Results}

Over the study period there were a total of 639 isolates identified as C. diphtheriae, where 638/639 (99\%) were from human disease and 1/639 (1\%) was from a horse. Twenty-two isolates ( $n=17 ; 77 \%$ from humans and $n=5 ; 23 \%$ from animals) were identified as $C$. ulcerans and no isolates were identified as C. pseudotuberculosis.

\section{Corynebacterium diphtheriae}

Between 2006 and 2012, an average of fourteen isolates of C. diphtheriae was referred annually. Between 2013 and 2015 (which coincided with the increasing use of MALDI-TOF), the number of annual referrals increased to an average of 40 . Between 2016 and 2018, the number of annual referrals increased to 115 . Between January and July 2019, an average of 15-16 referrals per month had been received at the NML: extrapolated, this would be approximately 185 by the end of the year. Comparing the pre-MALDI-TOF era for toxin testing of eight strains (in 2006) to an estimated 185 referrals projected for 2019 , this represented a $1,200 \%$ or 12 -fold increase over the review period (Figure 1).

Figure 1: Corynebacterium diphtheriae referrals for toxin testing by year, subset by number of toxigenic strains

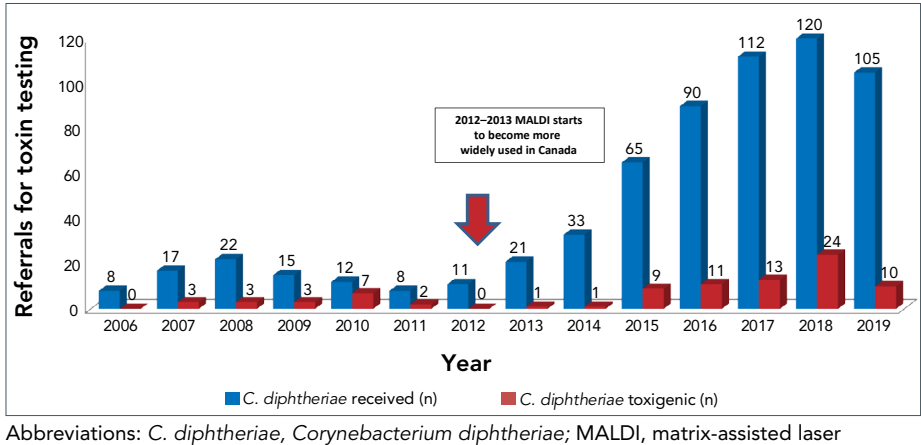
desorption/ionization spectrometry

Strains identified as $C$. diphtheriae were derived primarily from western Canada ( $n=609 / 639 ; 95 \%)$, that is, from British Columbia, Alberta, Saskatchewan and Manitoba, with small numbers from Ontario, Quebec and New Brunswick and from outside of Canada (Figure 2).

Figure 2: Corynebacterium diphtheriae and C. ulcerans referrals, by province

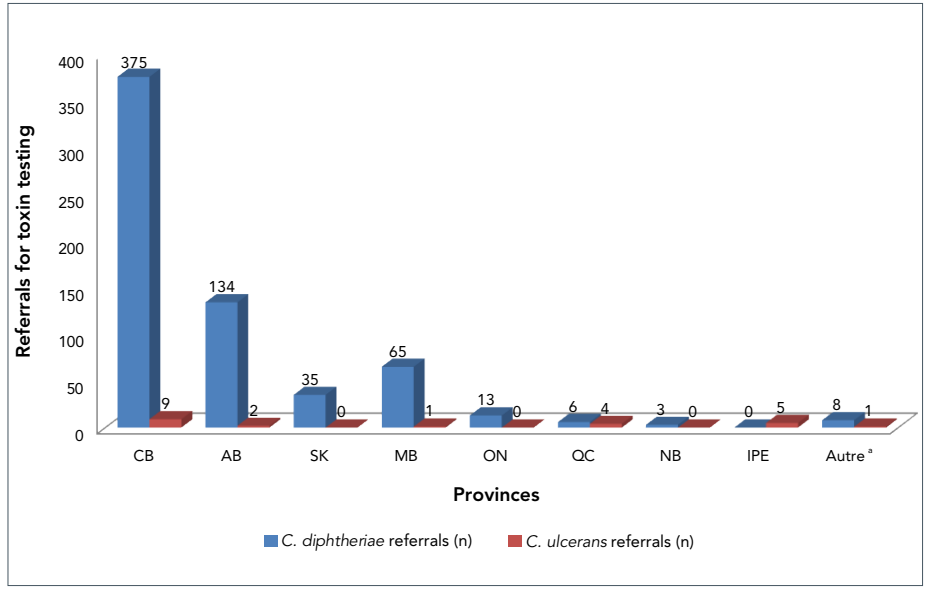

Abbreviations: AB, Alberta; BC, British Columbia; C., Corynebacterium; MB, Manitoba; NB, New Brunswick; ON, Ontario; PEI, Prince Edward Island; QC, Quebec; SK, Saskatchewan Other, sources outside of Canada

Referrals of $C$. diphtheriae subdivided by province were as follows (\% of $n=631$ ): British Columbia, 375 (59\%); Alberta, 134 (21\%); Saskatchewan, 35 (5\%); Manitoba, 65 (10\%); Ontario, 13 (2\%); Quebec, six (0.9\%); and New Brunswick, three $(0.4 \%)$. No C. diphtheriae referrals were received from Nova Scotia, Prince Edward Island or Newfoundland, and eight were received 
from outside of Canada. Referrals of C. ulcerans by province were as follows ( $\%$ of $n=21$ ): British Columbia, nine $(43 \%)$; Alberta, two (9\%); Manitoba, one (5\%); Quebec, four (19\%); and Prince Edward Island, five (24\%, all from animals). No strains were referred from Saskatchewan, Ontario, New Brunswick, Nova Scotia or Newfoundland, and one from outside of Canada.

The majority of the 105 C. diphtheriae strains from 2019 were obtained from skin infections. There were 89 (85\%) specimens from cutaneous sites that included the abdomen and abscesses/wounds from the ankle, arm, elbow, foot, finger, head/scalp, hip, leg, knee, shin, thigh, thumb, tibia and toe; 16 specimens (15\%) from non-cutaneous sites that included blood culture $(n=1)$, uterine $(n=1)$, ear $(n=2)$, sinus, sinus washing, nasal wound and nose $(n=5)$, throat $(n=4)$, sputum $(n=1)$ and unknown sites $(n=2)$.

In 2019, there were 10 toxigenic strains recovered from nose or cutaneous sites. These referrals were from Alberta $(n=8)$, British Columbia $(n=1)$ and Manitoba $(n=1)$. No patient was symptomatic of classic, respiratory-type diphtheria.

\section{Corynebacterium ulcerans}

A small number of referrals ( $n=22$, Figure 3 ) were identified as C. ulcerans. Seventeen (77\%) samples were obtained from human cases, with sources described as pus or from cutaneous sources (arm, elbow, foot, leg or toe wounds). Five animal-derived specimens were also received from mink ( $n=1$, lung), dog $(n=1$, ear), cat $(n=1)$ and horse ( $n=2$, abscess, skin). Such testing had also increased by approximately 35\% between 2006-2012 and 2013-2019 (Figure 3). Critically, although numbers were small, we found that $10 / 22$ (45\%) of C. ulcerans strains produced DT.

Figure 3: Corynebacterium ulcerans referrals for toxin testing by year, subset by number of toxigenic strains

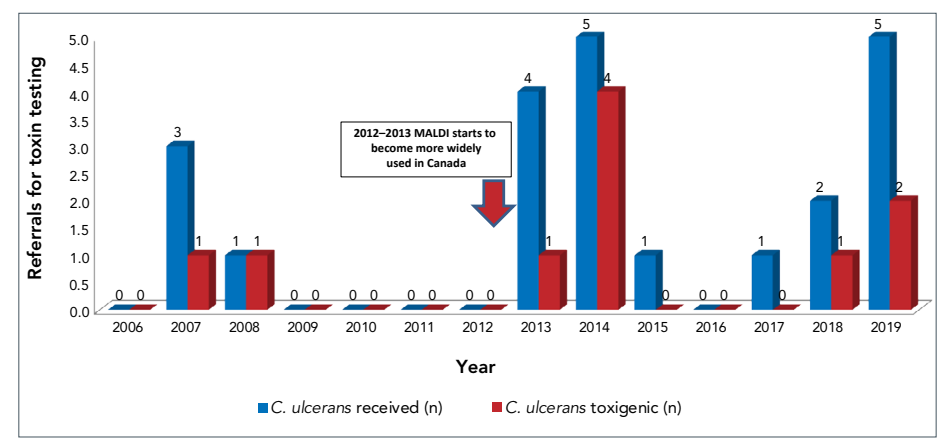

Abbreviations: C. ulcerans, Corynebacterium ulcerans; MALDI, matrix-assisted laser desorption/ionization spectrometry

Notes: Five C. ulcerans strains shown from 2019 were received from a veterinary laboratory. The remaining 17 samples were obtained from human sources

\section{Discussion}

The NML has documented a 1,200\% or 12-fold increase in referrals of Corynebacterium specimens for DT testing in less than 15 years. These are predominately non-toxigenic C. diphtheriae strains from cutaneous sources in humans recovered in pure culture or co-recovered in association with other Gram-positive bacteria (6). Although the overall numbers were small, a $35 \%$ increase of referrals involving C. ulcerans was found, that included strains from humans and animals. Similar increases of both $C$. diphtheriae and C. ulcerans have been reported in other countries including the United States (7-11). This increase is likely due in part to the increasingly widespread use of MALDI-TOF systems and precise molecular identification methods. It may also reflect that $C$. diphtheriae is an emerging pathogen for non-respiratory infections.

\section{Clinical implications}

Canadian clinicians should be aware that identification of C. diphtheriae and C. ulcerans from certain patient and specimen types is becoming increasingly common. Strains should be tested for toxin production and, if toxigenic, a public health response, including testing/monitoring of contacts of patients, may be indicated in addition to the usual treatment approaches (5).

Antibiotic sensitivity testing of these species has only rarely been requested but may be useful. In a recent article, we described antimicrobial susceptibility testing (AST) results based on an ancillary study of 195 strains of $C$. diphtheriae and 20 strains of C. ulcerans (22). Although these species are usually susceptible to first line antibiotics (22), the occasional strain of $C$. diphtheriae has been found to be penicillin or multidrug resistant $(13,23)$; in some instances, azithromycin may be indicated (10). Of note, the Canadian notifiable disease definition for diphtheria includes $C$. diphtheriae as well as both C. ulcerans and C. pseudotuberculosis and, if identified in a symptomatic patient, would be classified as a confirmed case (5). Cutaneous diphtheria would also be classified as a confirmed case if a toxigenic strain was recovered from a wound/cutaneous site (5).

\section{Limitations}

There are some inherent limitations to the results shown here. It is not mandatory for laboratories to send these taxa to the NML for further characterization. For example, Cadham Provincial Laboratory conducted their own DT testing by PCR on more than 600 strains of C. diphtheriae in the first six months of 2019all were found to be negative for the presence of tox genes (D Alexander, personal communication, July 24, 2019). These were not referred to the NML so the data presented here from the NML is an underestimate.

Identification of $C$. diphtheriae or $C$. ulcerans from a variety of clinical specimens, especially cutaneous sites, requires that local laboratories are able to select for/recognize these agents from among flora found in the biomes of infected cutaneous or respiratory sites. An algorithm may assist in the process of determining putative $C$. diphtheriae or $C$. ulcerans strains (1). Furthermore, it is difficult to conjecture if the lack of strains received for DT testing from central and eastern Canada reflects that no actual cases exist, or they occur more rarely than in the 
west. It may reflect the use of different guidance documents or different laboratory practices.

\section{Future research}

Ideally, the next steps could include both clinical and laboratory research, which would include assessment of cutaneous diphtheria as an emerging infection and collection of more complete clinical data on treatment versus outcome. Further laboratory research on these agents includes consensus-based development of a national and internationally-based typing scheme for characterization of such strains at whole genome sequence level, for use in silico tracking of strains and for better characterization of virulence factors.

\section{Conclusion}

In less than 15 years, there has been a marked increase in requests for DT testing of $C$. diphtheriae and $C$. ulcerans in Canada. This increase could be due to an enhanced ability to identify these agents using MALDI-TOF systems. Ongoing monitoring of these bacterial strains will help to assess whether the increase in NML referrals is due solely to increased precision of diagnosis or whether these are emerging cutaneous pathogens.

\section{Authors' statement}

$K A B$ wrote the manuscript and reviewed the data ALP, TB and DW performed all technical assays and reviewed the manuscript

\section{Conflict of interest}

None.

\section{Acknowledgements}

We gratefully acknowledge the following centres for sending study strains to the National Microbiology Laboratory: Dr. L Hoang, BC Centre for Disease Control, Vancouver, British Columbia; Dr. G Tyrell and S Gee, Provincial Laboratory, Edmonton, Alberta; R Kitzel, R Romanow Provincial Laboratory, Regina, Saskatchewan; Dr. P Van Caeseele and Dr. D Alexander, Cadham Laboratory, Winnipeg, Manitoba; Dr. J Kus, Public Health Ontario, Toronto, Ontario (ON); Dr. H Dick, Ideex Laboratories, Richmond Hill, ON; Dr. MC Domingo, Laboratoire de Santé Publique du Québec, Sainte-Anne-de-Bellevue, Quebec; Staff, the Moncton Hospital, Moncton,

New Brunswick (NB); Staff, Hôpital Georges-Dumont, Moncton, NB; M Saab, Atlantic Veterinary Hospital, Charlottetown, Prince Edward Island. We thank current and former students A Vachon, D Kielich and J Ung for technical services provided.

\section{Funding}

Public Health Agency of Canada A Base funding was used for this study.

\section{References}

1. Bernard KA. Coryneform Gram-Positive Rods. In: Carroll KC, Pfaller MA, Landry ML, McAdam AJ, Patel R, Richter SS, et al., editors. Manual of Clinical Microbiology. 12th ed. Washington, DC: ASM Press; 2019. p. 488-534.

2. Bernard KA, Funke G. Genus I. Corynebacterium Lehmann and Neumann 1896, 350AL emend. Bernard, Wiebe, Burdz, Reimer, Ng, Singh, Schindle and Pacheco 2010, 877. In: Whitman WB, Goodfellow M, Kämpfer P, Busse H-, Trujillo ME, Ludwig W, et al, editors. Bergeys Manual of Systematic Bacteriology. Volume 5: The Actinobacteria. 2nd ed. New York, NY: Springer; 2012. p. 245-89.

3. Bernard K. The genus corynebacterium and other medically relevant coryneform-like bacteria. J Clin Microbiol 2012 Oct;50(10):3152-8. DOI PubMed

4. Public Health Agency of Canada. Vaccine-Preventable Diseases-Diphtheria: For Health Professionals. PHAC; 2018. https://www.canada.ca/en/public-health/services/ immunization/vaccine-preventable-diseases/diphtheria/ health-professionals.html

5. Public Health Agency of Canada. National case definition: Diphtheria. Surveillance. PHAC; 2018. https://www.canada.ca/en/public-health/services/ immunization/vaccine-preventable-diseases/diphtheria/ health-professionals/national-case-definition.html

6. Lowe CF, Bernard KA, Romney MG. Cutaneous diphtheria in the urban poor population of Vancouver, British Columbia, Canada: a 10-year review. J Clin Microbiol 2011 Jul;49(7):2664-6. DOl PubMed

7. Griffith J, Bozio CH, Poel AJ, Fitzpatrick K, DeBolt CA, Cassiday P, Kenyon C, Smelser C, Vagnone PS, Culbreath K, Acosta AM. Imported Toxin-Producing Cutaneous Diphtheria - Minnesota, Washington, and New Mexico, 2015-2018. MMWR Morb Mortal Wkly Rep 2019 Mar;68(12):281-4. DOI PubMed

8. Meinel DM, Kuehl R, Zbinden R, Boskova V, Garzoni C, Fadini D, Dolina M, Blümel B, Weibel T, Tschudin-Sutter S, Widmer AF, Bielicki JA, Dierig A, Heininger U, Konrad R, Berger A, Hinic V, Goldenberger D, Blaich A, Stadler T, Battegay $M$, Sing $A$, Egli $A$. Outbreak investigation for toxigenic Corynebacterium diphtheriae wound infections in refugees from Northeast Africa and Syria in Switzerland and Germany by whole genome sequencing. Clin Microbiol Infect 2016 Dec;22(12):1003.e1-8. DOI PubMed

9. Reynolds GE, Saunders H, Matson A, O'Kane F, Roberts SA, Singh SK, Voss LM, Kiedrzynski T. Public health action following an outbreak of toxigenic cutaneous diphtheria in an Auckland refugee resettlement centre. Commun Dis Intell Q Rep 2016 Dec;40(4):E475-81. PubMed 
10. Morgado-Carrasco D, Riquelme-Mc Loughlin C, Fustá-Novell X, Fernández-Pittol MJ, Bosch J, Mascaró JM Jr. Cutaneous Diphtheria Mimicking Pyoderma Gangrenosum. JAMA Dermatol 2018 Feb;154(2):227-8. PubMed

11. Coen M, Alberto C, Éperon G, Cherkaoui A, Schrenzel J. [Diphtheria: new clinical presentations of an old disease]. Rev Med Suisse 2019 Apr;15(646):786-90. PubMed

12. de Benoist AC, White JM, Efstratiou A, Kelly C, Mann G, Nazareth B, Irish CJ, Kumar D, Crowcroft NS. Imported cutaneous diphtheria, United Kingdom. Emerg Infect Dis 2004 Mar;10(3):511-3. DOI PubMed

13. Mina NV, Burdz T, Wiebe D, Rai JS, Rahim T, Shing F, Hoang L, Bernard K. Canada's first case of a multidrug-resistant Corynebacterium diphtheriae strain, isolated from a skin abscess. J Clin Microbiol 2011 Nov;49(11):4003-5. DOI PubMed

14. Barberis $C$, Almuzara M, Join-Lambert $O$, Ramírez MS, Famiglietti A, Vay C. Comparison of the Bruker MALDI-TOF mass spectrometry system and conventional phenotypic methods for identification of Gram-positive rods. PLoS One 2014 Sep;9(9):e106303. DOI PubMed

15. Patel R. Matrix-assisted laser desorption ionization-time of flight mass spectrometry in clinical microbiology. Clin Infect Dis 2013 Aug;57(4):564-72. DOI PubMed

16. Khamis A, Raoult D, La Scola B. rpoB gene sequencing for identification of Corynebacterium species. J Clin Microbiol 2004 Sep;42(9):3925-31. DOI PubMed

17. Schuhegger R, Lindermayer M, Kugler R, Heesemann J, Busch U, Sing A. Detection of toxigenic Corynebacterium diphtheriae and Corynebacterium ulcerans strains by a novel real-time PCR. J Clin Microbiol 2008 Aug;46(8):2822-3. DOI PubMed
18. De Zoysa A, Efstratiou A, Mann G, Harrison TG, Fry NK. Development, validation and implementation of a quadruplex real-time PCR assay for identification of potentially toxigenic corynebacteria. J Med Microbiol 2016 Dec;65(12):1521-7. DOI PubMed

19. Engler KH, Glushkevich T, Mazurova IK, George RC, Efstratiou A. A modified Elek test for detection of toxigenic corynebacteria in the diagnostic laboratory. J Clin Microbiol 1997 Feb;35(2):495-8. PubMed

20. Brown C; Diphtheria Guidelines Working Group. Public health control and management of diphtheria (in England and Wales). 2015 Guidelines. London, UK: Public Health England; 2015. https://assets.publishing.service.gov.uk/ government/uploads/system/uploads/attachment_data/ file/774753/Diphtheria_Guidelines_Final.pdf

21. DeWinter LM, Bernard KA, Romney MG. Human clinical isolates of Corynebacterium diphtheriae and Corynebacterium ulcerans collected in Canada from 1999 to 2003 but not fitting reporting criteria for cases of diphtheria. J Clin Microbiol 2005 Jul;43(7):3447-9. DOI PubMed

22. Bernard KA, Pacheco AL. In vitro Activity of 22 Antimicrobial Agents against Corynebacterium and Microbacterium species referred to the Canadian National Microbiology Laboratory. Clin Microbiol Newsl 2015;37(23):187-98. DOI

23. FitzGerald RP, Rosser AJ, Perera DN. Non-toxigenic penicillin-resistant cutaneous $\mathrm{C}$. diphtheriae infection: a case report and review of the literature. J Infect Public Health 2015 Jan-Feb;8(1):98-100. DOI PubMed 